\title{
Ein Jahr Relaunch der markt - Journal für Marketing
}

\author{
Alexander Zauner
}

Online veröffentlicht: 12. Mai 2010

(C) Springer-Verlag 2010

Mit Anfang 2009 wurde von den Herausgebern ein Relaunch der traditionsreichen Zeitschrift der markt in Kooperation mit dem Springer-Verlag durchgeführt. Nicht nur der Titel hat sich auf der markt - Journal für Marketing geändert, sondern es wurde u. a. ein Editorial Board mit international anerkannten Marketing-Wissenschaftlern und ein webbasiertes Reviewing-Systems aufgebaut.

Seitdem wird ergänzend zur Berücksichtigung der Besonderheiten des deutschsprachigen Raumes, die oftmals über Erfolg oder Misserfolg von Marketing in diesem Kulturkreis entscheiden, der Fokus verstärkt auf eine gesamteuropäische Perspektive gelegt. Ein besonderes Anliegen ist und bleibt die Offenheit für die Vielfalt wissenschaftlicher Blickwinkel und methodischer Zugänge. der markt möchte einen Beitrag dazu leisten, innovativen und alternativen Denkansätzen, die den Mainstream der Forschung verlassen, zum Durchbruch zu verhelfen. Rasches Aufgreifen aktueller Themen, neue Ansätze und Erkenntnisse für die Marketing-Praxis machen die Zeitschrift zu einer Diskussionsplattform für national und international renommierte Autoren. Die vielen positiven Rückmeldungen und die große Anzahl von qualitativen Einreichungen unterstreichen den Erfolg dieser Neuausrichtung.

Alle Manuskripte werden zunächst von den Herausgebern in Zusammenarbeit mit dem wissenschaftlichen Beirat auf ihre grundsätzliche Eignung überprüft und anschließend von mindestens zwei internationalen Experten „double-blind“ begutachtet, d. h. bewertet und konstruktiv

\section{A. Zauner (}

WU Wien,

Department für Marketing,

Institut für Marketing-Management,

Augasse 2-6, 1090 Wien, Österreich

E-Mail: alexander.zauner@wu.ac.at weiterentwickelt. Im ersten Jahrgang nach dem Relaunch konnten auf diese Weise zwölf Beiträge von höchster Qualität publiziert werden.

Mit dem vorliegenden Heft starten wir in ein hoffentlich ebenso erfolgreiches zweites Jahr. In den Ausgaben Nr. 1 und 2 des 49. Jahrgangs werden vielfältigste Manuskripte und Themen mit Marketingbezug wissenschaftlich aufgearbeitet und besprochen. Im Herbst 2010 wird ein Special Issue zu „Neuromarketing und apparativen Messmethoden“ erscheinen. Frau Dr. Monika Koller, WU Wien, wird dieses Thema als Special Issue Editor betreuen. In diesem Sinne möchte ich auch auf den Call for Papers zu ,Loyalty Programs von Banken und Versicherungen“ hinweisen. Herr Prof. Dr. Ewald Judt, WU Wien, wird dieses wissenschaftlich hoch-relevante Thema in einer für 2011 vorgesehenen Schwerpunktausgabe behandeln.

Ich möchte diese Gelegenheit nutzen, um mich ganz herzlich bei allen Autoren und Gutachtern für deren wertvollen Beitrag zum Gelingen des ersten Jahrgangs zu bedanken. Besonderer Dank gebührt auch unseren Beiräten, der Redaktion und Herrn Prof. Dr. Fritz Scheuch als Schirmherr von der markt - Journal für Marketing.

Abschließend darf nicht unerwähnt bleiben, dass die Österreichische Gesellschaft für Absatzwirtschaft (ÖGA) von namhaften Unternehmen und Organisationen aus der Wirtschaft unterstützt wird. Ohne deren Förderung könnten die ambitionierten Ziele nicht verfolgt werden. Wir bedanken uns insbesondere bei folgenden Unternehmen und Organisationen: Baumax AG, BAWAG-PSK, Casinos Austria AG, Feibra Werbung Gesellschaft m.b.H., Henkel Austria Gesellschaft m.b.H., Kelly Gesellschaft m.b.H., Unilever Austria GmbH, mobilkom austria AG, Finanzmarketing-Verband Österreich, Wiener Städtische Versicherung, SPAR Österreich Warenhandels AG, Vöslauer Mineralwasser AG, ZEV Markant Zentrale Einkaufs- 
und Vertriebsgesellschaft m.b.H. und Mittelstandskreis Handels-GmbH.

Ihr

Alexander Zauner 